\title{
Guest Editorial: Wavelets in Medical Imaging
}

\section{INTRODUCTION}

W AVELETS are the result of collective efforts that recognized common threads between ideas and concepts that had been independently developed and investigated by distinct research communities. They provide a unifying framework for decomposing images, volumes, and time-series data into their elementary constituents across scale. Although a relatively recent construct, wavelets have become a tool of choice for engineers, physicists, and mathematicians, leading to efficient solutions in time and space frequency analysis problems, as well as a multitude of other applications. One of the consequences is that wavelet methods of analysis and representation are presently having a significant impact on the science of medical imaging and the diagnosis of disease and screening protocols. Because of a powerful underlying mathematical theory, they offer exciting opportunities for the design of new multiresolution image processing algorithms, and novel acquisition methods such as wavelet-encoded magnetic resonance imaging (MRI). This special issue of the IEEE TRANSACTIONS on Medical Imaging focuses on these recent developments and highlights progress that has been accomplished in the areas related to medical imaging.

\section{SizING THE WaVE: Some FACTS AND FiguRES}

Rarely has a mathematical concept generated so much response and enthusiasm within and between the engineering and mathematical research communities at large. To give a rough idea of the phenomenon, we provide a brief chronology. While wavelets have been traced all the way back to Alfred Haar in 1910 [1], for many, the starting point of their modern history coincides with two publications in the late 1980s by S. Mallat [2] and I. Daubechies [3]. These groundbreaking papers established a solid mathematical footing which would both shape and define the field. In a nutshell, S. Mallat identified the important concept of multiresolution analysis which is the corner stone of modern wavelet theory, while I. Daubechies constructed the first orthogonal wavelet bases that were compactly supported. These two contributions count among the most cited papers in the scientific literature (over 1500 SCI citations each). From that point on, the number of contributions relating to wavelet-applications and theory has increased steadily on the order of 9000 journal papers published to date. This trend is likely to continue as suggested by the strong response to the call for papers for this special issue (over 30 submissions).

Wavelets have become so popular that distinct communities continue to have conferences and scientific journals entirely de-

Digital Object Identifier 10.1109/TMI.2003.809638 voted to them. There are already historical anecdotes and folklore associated with them; an entertaining account of which can be found in the book of B. Burke Hubbard [4]. Readers who want to dive deeper into the subject have the daunting task of choosing among over 200 books written on wavelets. Our only advice in this regard is: in case of doubt, stick with the classics.

Given the size of the phenomenon, it is no surprise that wavelets have had an impact on a number of disciplines, medical imaging being no exception. A first record of activity in this particular area is the workshop on wavelets in medicine and biology that took place at the annual IEEE-EMBS meeting, Baltimore, MD, 1992. The first journal paper describing a wavelet application in medical imaging-noise reduction in MRI by soft-thresholding in the wavelet domain-also appeared in 1992 [5]. Note that this work, which is often overlooked, provides the earliest description of a wavelet denoising method that has become extremely popular through the impulsion of Donoho et al. A large palette of wavelet applications in medical imaging is provided in [6]. Two complementary review articles are also available; the first gives a complete account of the activity taking place from the beginning to 1996 [7], while the second covers the more recent papers until 2000 [8]. So far, the primary applications of wavelets in medical imaging have been the following:

- Compression of medical images.

- CT reconstruction; local tomography.

- Wavelet denoising (MRI, ultrasound).

- Wavelet-based feature extraction; texture and statistical descriptors

- Medical image enhancement (e.g., fluoroscopy and mammography).

- Analysis of functional images of the brain [positron emission tomography (PET), functional MRI (fMRI)].

- Wavelet-encoded MRI.

Most of these topics are still active areas of research, as illustrated by the papers that are published in this special issue.

\section{SCANNING THROUGH THE ISSUE}

Functional imaging is an area were methods of wavelet processing hold great promise. This particular line of research was initiated by U. Ruttimann, a creative researcher and good friend, who sadly passed away shortly before the publication of his paper in this very journal [9]. Another first rate statistician who was also active in this area at an early stage is J. Raz. By a sad coincidence, he also suffered a sudden death about a week before he was to present his latest results on wavelet analysis of fMRI [10]. Despite the tragic loss of these two pioneers, research in this area is alive and well as exemplified by the first three papers of this issue. Turkheimer et al. [11] consider the problem 
of the analysis of dynamic PET data; in particular, they advocate the use of a linear (James-Stein) wavelet estimator as an alternative to the more classical wavelet shrinkage or threshold detectors. Hossein-Zadeh et al. propose a wavelet-technique for the detection of activation in fMRI data [12]. Their contribution is twofold: first, the use of a redundant wavelet transform for better translation invariance, and second, a nonparametric detection method based on a randomization procedure. F. Meyer also considers fMRI time series but applies wavelets differently, within the context of a generalized linear model, to detrend the data [13]; that is, to get rid of signal drifts and disturbances that are not related to the stimulus.

Two other areas where wavelets have achieved great success is signal denoising (typically, by simple thresholding in the wavelet domain) and tomographic reconstruction, mainly, because the Radon operator is well localized in a wavelet basis. Pizurica et al. [14] propose a novel wavelet denoising method that uses a local statistical model for improved signal estimation and noise suppression. Willett and Nowak [15] introduce a new multiscale image model, using piecewise planar basis functions, and apply their method to the reconstruction of photon-limited data (with Poisson noise). They develop penalized maximum likelihood methods for image denoising, deconvolution, and tomographic reconstruction. Kalifa et al. present a direct method for the efficient reconstruction of PET and single photom emission computed tomography data [16]. Their approach includes a nonlinear noise reduction step that is implemented by thresholding in the transformed domain; the key here is to select a transform (wavelet packet) that is optimized for the problem and data at hand (sparse representation of the signal and near diagonalization of the Radon operator). Bonnet et al. [17] also develop a direct approach for the reconstruction of cone-beam data which is known to be challenging. In essence, their approach is a wavelet adaptation of the Feldkamp algorithm.

The special issue also features two contributions relating to ultrasound imaging. Michailovich and Adam consider the problem of the estimation of the spectrum of a ultrasound pulse [18]. Specifically, they develop a modified (outlier-resistant) wavelet estimator that they apply to the log-spectrum of the radio-frequency sequence. Lee et al. present a pattern recognition system that uses an M-band wavelet filterbank to extract fractal and texture features from ultrasonic images of the liver [19]. They report promising classification results, differientiating normal liver, cirrhosis, and hepatoma using a hierarchical classifier.

The recent development of commercial digital mammography imaging systems not only provides a significant improvement in image quality for traditional screening, but translates into a wealth of information for the analysis and detection of mammographic features by computer. The papers by Lemaur et al. [20]. and Heinlein et al. [21] focus on the goals of early detection and visual enhancement of microcalcifications, respectively. In the former, the regularity of a wavelet basis is used to identify microcalcification in clusters. The identification of microcalcifaction in clusters as opposed to individual occurrences is of clinical significance as clusters may suggest the likelihood of malignancy. In the later paper, a discretization of the continuous wavelet transform is developed which allows a filterbank to be adapted for the enhancement of mammographic features. This implementation allows for the reconstruction of modified wavelet coefficients at arbitrary scales and orientations without the introduction of artifacts or loss of completeness. The integration of such an interactive enhancement tool into digital mammographic screening systems will be of great importance as the wealth of dynamic range (contrast) provided by digital detectors become generally available to radiologist through the introduction of lower cost softcopy display systems.

The paper of Davatzikos et al. [22] offers another illustration of the versatility of wavelets. Their proposal is to represent the contours of a shape in a wavelet bases and to use this sparse representation to derive active shape models. Their results are promising and significant in terms of providing an automated solution to problems in volume quantification.

The amount of data generated by modern imaging devices is often very large, and ever increasing. Thus, an important problem is to find efficient ways of compressing and encoding this information to facilitate its transmission, storage, and retrieval. Since wavelet transforms provide sparse representations of signals, one of their privileged areas of application has been coding and compression. In fact, wavelets have had so much success in this area that they have already become an integral part of the new JPEG2000 compression standard. Unlike other applications, however, biomedical image compression raises delicate issues; lossy compression must be done carefully so as to preserve all medically relevant information while eventually suppressing irrelevant features such as noise or background. Thus, research in this area must address these specific needs and pay great attention to the issue of validation. The paper by Menegaz and Thiran [23] uses wavelet transform techniques for encoding 3D volumetric data sets to optimally compress the volumetric images while allowing efficient two-dimensional slices reconstruction. Schelkens et al. [24] propose new wavelet methods for coding volumetric medical datasets, and compares them to state-of-the-art compression techniques. The new methods perform favorably for both lossy and lossless compression. The paper of Z. Xiong et al. [25] also proposes a new method of wavelet compression that is competitive with other state-of-the-art compression techniques but suggests improved image quality.

\section{CONCLUSION}

After having completed the tasks of putting this issue together and based on our collective knowledge in the field, it 
is our belief that wavelets are here to stay. The idea of decomposing a signal according to scale is as fundamental a notion for imaging as decomposing it into harmonic components (Fourier analysis); it is more intuitive and closer to what the visual system does. Another important point that has stimulated wavelet research is the quest for sparse representations of signals, as these offer obvious advantages for data compression, noise reduction and regularization purposes. As more progress is made in the years ahead, it is quite possible that the importance of wavelets as a research topic in its own right may diminish and that they will progressively reach the status of standard toolbox components for data processing (such as the fast Fourier transform). Indeed, we are seeing some of this maturity in the development of emerging commercial image analysis software and packages. Yet, given the flexibility of this powerful analysis tool, there is not much risk in betting that wavelets will continue to be widely used in a diversity of medical imaging applications.

A beneficial side effect of all the interdisciplinary activity taking place around wavelets has been to create a common language between mathematicians and engineers and to instill rigor in the formulation of imaging problems. Wavelets have been a cross-cutting magnet encouraging engineers to use more sophisticated mathematics and mathematicians to consider applying their knowledge to real world problems.

As Guest Editors, we hope that the papers in this issue will stimulate further progress in this direction. We believe that the best is yet to come. We are particularly grateful to the authors for their timely contributions and to the reviewers who responded quickly with insightful and thorough comments. We would also like to thank K. Escher for her editorial assistance and patience. Finally, we want to close by dedicating this special issue to U. Ruttimann and J. Raz.

\section{MiCHAEL UNSER, Guest Editor Swiss Federal Institute of Technology Biomedical Imaging Group EPFL LIB Lausanne, CH-1015 Switzerland \\ AKRAM ALDROUBI, Guest Editor Vanderbilt University Department of Mathematics Nashville, TN 37209 \\ ANDREW LAINE, Guest Editor Columbia University Department of Biomedical Engineering New York, NY 10027}

\section{REFERENCES}

[1] A. Haar, "Zur theorie der orthogonalen funktionensysteme," Math. Ann., vol. 69 , pp. 331-371, 1910 .
[2] S. G. Mallat, "A theory of multiresolution signal decomposition: The wavelet representation," IEEE Trans. Pattern Anal. Machine Intell., vol. PAMI-11, pp. 674-693, 1989.

[3] I. Daubechies, "Orthogonal bases of compactly supported wavelets," Commun. Pure Appl. Math., vol. 41, pp. 909-996, 1988.

[4] B. B. Hubbard, The World According to Wavelets. Wellesley, MA: A K Peters, 1998.

[5] J. B. Weaver, X. Yansun, D. M. Healy Jr, and L. D. Cromwell, "Filtering noise from images with wavelet transforms," Magn. Reson. Med., vol. 21, pp. 288-95, 1991.

[6] A. Aldroubi and M. Unser, Wavelets in Medicine and Biology. Boca Raton, FL: CRC, 1996.

[7] M. Unser and A. Aldroubi, "A review of wavelets in biomedical applications," Proc. IEEE, vol. 84, pp. 626-638, 1996.

[8] A. Laine, "Wavelets in temporal and spatial processing of biomedical images," Аnnu. Rev. Biomed. Eng., vol. 2, pp. 511-550, 2000.

[9] U. E. Ruttimann, M. Unser, R. R. Rawlings, D. Rio, N. F. Ramsey, V. S. Mattay, D. W. Hommer, J. A. Frank, and D. R. Weinberger, "Statistical analysis of functional MRI data in the wavelet domain," IEEE Trans. Med. Imag., vol. 17, pp. 142-154, Apr. 1998.

[10] J. Raz, "FDR wavelet shrinkage estimators for inhomogeneous poisson processed," presented at the SPIE Conf. Wavelets Applications in Signal and Image Processing VIII, San Diego, CA, 2000.

[11] F. E. Turkeimer, J. A. D. Aston, R. B. Banati, C. Riddell, and V. J. Cunningham, "A linear wavelet filter for parametric imaging with dynamic PET," IEEE Trans. Med. Imag., vol. 22, pp. 289-301, Mar. 2003.

[12] G.-A. Hossien-Zadeh, H. Soltanian-Zadeh, and B. A. Ardekani, "Multiresolution fMRI activation detection using translation invariant wavelet transform and statistical analysis based on resampling," IEEE Trans. Med. Imag., vol. 22, pp. 302-314, Mar. 2003.

[13] F. G. Meyer, "Wavelet based estimation of a semi parametric generalized linear model of fMRI time-series," IEEE Trans. Med. Imag., vol. 22, pp. 315-322, Mar. 2003.

[14] A. Pizurica and W. Philips, "A versatile wavelet domain noise filtration technique for medical imaging," IEEE Trans. Med. Imag., vol. 22, pp. 323-331, Mar. 2003.

[15] R. Willett and R. Nowak, "Platelets: A multiscale approach for recovering edges and surfaces in photon limited medical imaging," IEEE Trans. Med. Imag., vol. 22, pp. 332-350, Mar. 2003.

[16] J. Kalifa, A. Lain, and P. D. Esser, "Regularization in tomographic reconstruction using thresholding estimators," IEEE Trans. Med. Imag., vol. 22, pp. 351-359, Mar. 2003.

[17] S. Bonnet, F. Peyrin, F. Turjman, and R. Prost, "Nonseparable waveletbased cone-beam reconstruction in 3-D rotational angiography," IEEE Trans. Med. Imag., vol. 22, pp. 360-367, Mar. 2003.

[18] O. Michailovich and D. Adam, "Robust estimation of ultrasound pulses using outlier-resistant de-noising," IEEE Trans. Med. Imag., vol. 22, pp. 368-381, Mar. 2003.

[19] W.-L. Lee, Y.-C. Chen, and K.-S. Hsei, "Ultrasonic liver tissues classification by fractal feature vector based on M-band wavelet transform," IEEE Trans. Med. Imag., vol. 22, pp. 382-392, Mar. 2003.

[20] G. Lemaur, K. Drouiche, and J. DeConinck, "Highly regular wavelets for the detection of clustered microcalcifications in mammograms," IEEE Trans. Med. Imag., vol. 22, pp. 393-401, Mar. 2003.

[21] P. Heinlein, J. Drexl, and W. Schneider, "Integrated wavelets for enhancement of microcalcifications in digital mammography," IEEE Trans. Med. Imag., vol. 22, pp. 402-413, Mar. 2003.

[22] C. Davatzikos, X. Tao, and D. Shen, "Hierarchical active shape models, using the wavelet transform," IEEE Trans. Med. Imag., vol. 22, pp. 414-423, Mar. 2003.

[23] G. Menegaz and J.-P. Thiran, "3D encoding/2D decoding of medical data," IEEE Trans. Med. Imag., vol. 22, pp. 424-440, Mar. 2003.

[24] P. Schelkens, A. Munteanu, J. Barbarien, M. Galca, X. G. I. Nieto, and J. Cornelis, "Wavelet coding of volumetric medical datasets," IEEE Trans. Med. Imag., vol. 22, pp. 441-458, Mar. 2003.

[25] Z. Xiong, X. Wu, S. Cheng, and J. Hua, "Lossy-to-lossless compression of medical volumetric data using three-dimensional integer wavelet transforms," IEEE Trans. Med. Imag., vol. 22, pp. 459-470, Mar. 2003. 


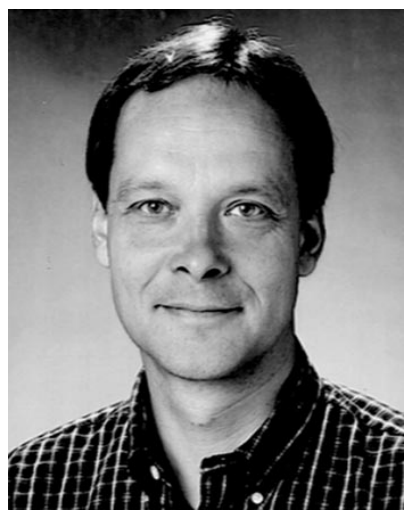

Michael Unser (M'89-SM'94-F'99) received the M.S. (summa cum laude) and Ph.D. degrees in electrical engineering from the Swiss Federal Institute of Technology, Lausanne (EPFL), Switzerland, in 1981 and 1984, respectively.

From 1985 to 1997, he was with the Biomedical Engineering and Instrumentation Program, National Institutes of Health, Bethesda, MD, where he headed the Image Processing Group. He is now Professor and Head of the Biomedical Imaging Group at the EPFL. His main research area is biomedical image processing. He has a strong interest in sampling theories, multiresolution algorithms, wavelets, and the use of splines for image processing. He is the author of 100 published journal papers in these areas.

Since January 2003, Dr. Unser is Associate Editor-in-Chief for the IEEE TRANSACTIONS ON MEDICAL IMAGING. He is also on the editorial boards of several other journals including the IEEE Signal Processing Magazine. He was former Associate Editor for the IEEE TRANSACTIONS ON IMAge Processing (1992-1995) and the IEEE SignAl Processing LeTTERS (1994-1998). He co-organized the IEEE workshop on Wavelets in Medicine and Biology in 1992. He serves as regular co-chair for SPIE's conference on wavelets, which has been held annually since 1993. He was general co-chair for the first IEEE International Symposium on Biomedical Imaging (ISBI'2002), Washington, DC, July 7-10, 2002.

He received the Dommer prize for excellence from the Swiss Federal Institute of Technology in 1981, the research prize of the Brown-Boveri Corporation (Switzerland) for his thesis in 1984, the IEEE Signal Processing Society’s 1995 Best Paper Award, and IEEE Signal Processing Society's 2000 Magazine Award.

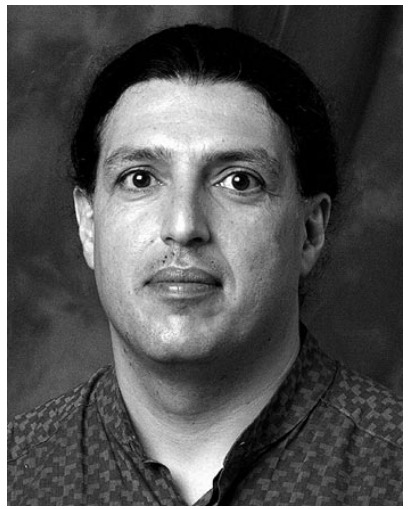

Akram Aldroubi received the Diplome in electrical engineering from the Swiss Federal Institute of Technology, Lausanne, Switzerland in 1982. He received the M.S. and Ph.D. degrees in mathematics from Carnegie-Mellon University, Pittsburgh, PA, in 1984 and 1987, respectively.

He is currently a Professor of Mathematics at Vanderbilt University, Nashville, TN. His prior position was with the National Institutes of Health. He has held a visiting position at the CNRS, France, in 1997. His research interest includes sampling theory, frames, wavelet theory, signal, and image processing of biomedical data.

Prof. Aldroubi is a member of the American Mathematical Society. He received the Rene Cousin Prize for excellence from the Swiss Federal Institute of Technology in 1982, the IEEE Signal Processing Society's 1995 Best Senior Paper Award (IMDSP technical area) for a Transaction paper (with M. Unser and M. Eden) on B-spline signal processing, and the Special Achievement Award of the National Center for Research Resources, at the National Institutes of Health in 1996. He is co-editor (with M. Unser) of the book Wavelet in Medicine and Biology (Boca Raton, FL: CRC, 1996), and co-editor (with E. B. Lin) of "Wavelets, multiwavelets, and applications," in Contemporary Mathematics (Providence, RI: American Mathematical Society, 1998).

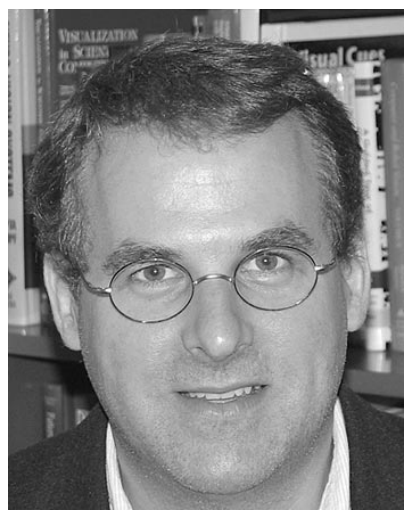

Andrew Laine received the D.Sc. degree from the School of Engineering and Applied Science in Computer Science, Washington University, St. Louis, MO, in 1989 and the B.S. degree from Cornell University, Ithaca, NY.

He was a Professor in the Department of Computer and Information Sciences and Engineering at the University of Florida, Gainesville, from 1990-1997. For the past five years, he has been the Director of the Biomedical Imaging Laboratory in the Department of Biomedical Engineering at Columbia University, New York City. He holds a joint appointment as Associate Professor of Biomedical Engineering and Radiology. He pioneered the application of multiresolution representations for feature analysis of digital mammography and ultrasound. His research interests include methods of multiresolution analysis applied to problems in medical imaging, image processing, computed aided diagnosis, pattern recognition, and applied mathematics.

Professor Laine served Associate Editor of IEEE TRANSACTIONS ON IMAGE PROCESSING and edited the book, Wavelet Theory and Applications (Norwell, MA: Kluwer, 1995). He has jointly chaired the conference on "Mathematical Imaging: Wavelet Application in Signal and Image Processing", during the years 19932003, and co-authored chapters in the books Wavelet Applications in Medicine and Biology (Boca Raton, FL: CRC Press, 1995) and Time-Frequency and Wavelets Transforms in Biomedical Engineering (Piscataway, NJ: IEEE Press, 1998). He is a member of the Editorial Board of the book series Emerging Technologies in Biomedical Engineering, sponsored by the IEEE-EMBS Society. He also served on the program committee for the IEEE-EMBS Workshop on Wavelet Applications in Medicine in 1994, 1998 and 1999. He is an active member of the IEEE Computer Society and Engineering in Medicine and Biology Society. 